\title{
Adrenal Cortisol Hyper-Responsiveness as a Possible Cause of Obesity, Insulin Resistance and Cardiovascular Risk
}

\author{
Yadon Arad ${ }^{1,2 *}$, Simcha Pollack ${ }^{1,3}$, Marguerite Roth $^{1}$ and Alan D Guerci ${ }^{1}$ \\ ${ }^{1}$ St Francis hospital, Roslyn, NY, USA \\ ${ }^{2}$ Tiara Pharmaceuticals, Los Altos, CA, USA \\ ${ }^{3}$ St John's University, Queens, NY, USA
}

*Corresponding author: Yadon Arad MD, CEO, Tiara Pharmaceuticals, 4546 B10 El Camino Real, \#304, Los Altos CA 94022, USA, Tel: 917-817-4757; E-mail: yadonarad@gmail.com

Rec date: Apr 23, 2014; Acc date: Sep 23, 2015; Pub date: Sep 28, 2015

Copyright: (c) 2015 Arad Y, et al. This is an open-access article distributed under the terms of the Creative Commons Attribution License, which permits unrestricted use, distribution, and reproduction in any medium, provided the original author and source are credited.

\begin{abstract}
Introduction: Increased cortisol concentrations due to endogenous over-secretion or exogenous administration result in obesity, insulin resistance, and increased risk of cardiovascular disease (CVD). We wanted to assess whether non pathological adrenal hyper responsiveness to ACTH could reproduce these changes and thus explain how stress, via the pituitary-adrenal axis, can also cause such abnormalities.
\end{abstract}

Methods: ACTH stimulation test was performed on 1072 non diabetic healthy participants in the St. Francis Heart Study and multiple blood tests, anthropometric measurements and coronary calcium scores were also obtained.

Results: Post stimulation cortisol concentrations were correlated with obesity, insulin resistance, lipid abnormalities, Framingham scores (all $p \leq 0.01$ ), but not with blood pressure and not with coronary calcium scores. In multivariate models, increased cortisol secretion was significantly predictive of abdominal fat even after controlling for insulin resistance, and of insulin resistance even after controlling for abdominal fat.

Conclusions: Adrenal hyper responsiveness may explain some of the observation of the relationship between stress and the metabolic abnormalities which can lead to CVD. Increased cortisol secretion predicts insulin resistance and obesity, independently of each other.

Keywords: Cortisol; Glucose; Insulin; Obesity; Lipids; ACTH; Adrenal glands; HOMA

\section{Introduction}

Insulin resistance, central adiposity, hypertension and lipid abnormalities, sometimes combined in the term "the metabolic syndrome" [1-3], predispose to future risk of diabetes mellitus type II and are independent risk factors for cardiovascular disease [4]. The cause(s) of the metabolic syndrome and its components is unknown. Many of the metabolic abnormalities resemble a milder form of the metabolic derangements seen in Cushing's syndrome. However, almost none of these patients have Cushing's syndrome. Since stress is speculated to be a mediator of CVD [5-7], and stress is associated with increased cortisol secretion, and since exogenous cortisol also results in similar metabolic derangements, it is possible that excess secretion of cortisol in response to stimuli, including stress, while in levels lower than those seen in frank Cushing's syndrome, may be a mediator of these metabolic abnormalities.

\section{Methods}

The St. Francis Heart Study was a multi-modality study incorporating a natural history arm, a randomized clinical trial and a risk factor analysis study. The design of the study was previously published [8]. Briefly, 5000 subjects underwent screening for coronary disease using electron beam CT to measure coronary artery calcium (CAC). Those subjects with a high score (defined as greater than $80 \%$ for age and gender adjusted scores) were enrolled in a randomized clinical trial on the effect of combination therapy with atorvastatin, vitamin $\mathrm{C}$ and vitamin $\mathrm{E}$ versus placebo on cardiac events and CAC progression [9]. Both placebo and intervention groups took $81 \mathrm{mg}$ of aspirin a day. Those with lower scores and the placebo arm of the RCT also took part in the natural history sub study [10].

The risk factor sub study: One fifth of the subjects from the low CAC score group and one third of the subjects from the high CAC score group were randomly selected to participate in the risk factor sub study. This generated a more or less continuous distribution of patients among the CAC score distribution spectrum with a total of 1167 subjects of whom 1072 completed this study. This population then underwent a series of baseline measurements and physiologic testing as follows:

Oral glucose tolerance test: serum samples were tested for glucose and insulin at base line and one hour after ingestion of 100 gram of glucose.

A combination cortisol suppression and stimulation test: For this test the subjects were administered $1 \mathrm{mg}$ of dexamethasone orally at 11 $\mathrm{pm}$ on the night before the test. The following morning at $8 \mathrm{AM}$ samples were collected for suppressed cortisol concentration. Immediately after, $250 \mathrm{mcg}$ of ACTH was administered intravenously 
Citation: Arad Y, Pollack S, Roth M, Guerci AD (2015) Adrenal Cortisol Hyper-Responsiveness as a Possible Cause of Obesity, Insulin Resistance and Cardiovascular Risk. J Diabetes Metab 6: 608. doi:10.4172/2155-6156.1000608

Page 2 of 5

and samples were collected again one hour later. The purpose of this test was to isolate adrenal response to stimulated stress and achieve a uniform base line for all subjects. Measurements were taken of TG, TC, LDL, apolipoprotein B and HDL.

\section{Systolic and diastolic blood pressure}

Measurements of body fat were obtained via multiple parameters. Intra-abdominal fat and extra abdominal fat and abdominal height were obtained via a single CT slice at the level of the umbilicus with the subject in the supine position [11]. To obtain the quantity of intraabdominal fat and extra-abdominal fat the areas of fat $(<30 \mathrm{HU})$ were delineated on the image and summed up. Abdominal height was the distance between a horizontal line touching the anterior most part of the abdomen and the level of the table 1 . BMI was calculated as height (in $\mathrm{cm}$ ) / (weight (in kg))2.

\begin{tabular}{|c|c|c|c|}
\hline & 1st tertile post-stimulation cortisol & 3rd tertile post-stimulation cortisol & p-value \\
\hline Variable & mean (std dev) & mean (std dev) & \\
\hline FRAMINGHAM SCORE & $13.77(2.67)$ & $15.02(2.8)$ & $<.0001$ \\
\hline TOTAL CHOLESTEROL & $215.01(37.18)$ & $230.91(39.06)$ & $<.0001$ \\
\hline EXTRA ABDOMINAL FAT & $221.2(113.25)$ & $267.49(131.34)$ & $<.0001$ \\
\hline QUICKI & $0.15(0.01)$ & $0.14(0.01)$ & $<.0001$ \\
\hline GLU & $103.88(17.25)$ & $111.73(33.15)$ & $<.0001$ \\
\hline HOMA IR & $2.99(2.29)$ & $3.89(3.53)$ & $<.0001$ \\
\hline APOB & $109.23(23.63)$ & $119.24(24.25)$ & 0.0002 \\
\hline HDL & $49.48(13.05)$ & $53.48(16.14)$ & 0.0002 \\
\hline BASELINE INSULIN & $12.39(8.46)$ & $15.43(12.6)$ & 0.0002 \\
\hline INTRA ABDOMINAL FAT & $117.99(65.82)$ & $139.29(86.74)$ & 0.0002 \\
\hline WAIST CIRCUMFERENCE & $0.41(0.49)$ & $0.61(0.49)$ & 0.0037 \\
\hline BASELINE GLUCOSE & $94.65(13.87)$ & $98.37(21.1)$ & 0.0068 \\
\hline POSTSTIMULATION GLUCOSE & $109.74(44.18)$ & $120.1(56.04)$ & 0.0076 \\
\hline BMI & $28.05(4.56)$ & $28.97(4.93)$ & 0.0090 \\
\hline TG & $133.46(89.22)$ & $155.35(146.85)$ & 0.0148 \\
\hline CRP & $3.05(5.1)$ & $4.11(6.25)$ & 0.0160 \\
\hline POSTSTIMULATION INSULIN & $73.86(74.18)$ & $86.68(73.36)$ & 0.0236 \\
\hline AB HEIGHT & $25.08(3.69)$ & $25.59(4.22)$ & 0.0855 \\
\hline SBP & $135.49(19.3)$ & $137.17(20.65)$ & 0.2551 \\
\hline DBP & $78.47(9.2)$ & $78.22(10.04)$ & 0.7262 \\
\hline 4 YEAR CAC & $360.08(648.74)$ & $335.55(626.46)$ & 0.7323 \\
\hline BASELINE CAC & $217.49(450.37)$ & $208.24(408.47)$ & 0.7701 \\
\hline 2 YEAR CAC & $267.39(540.57)$ & $260.18(687.62)$ & 0.8873 \\
\hline
\end{tabular}

Table 1: The values of the variables studied are shown in relation to the tertiles of cortisol secretion in response to ACTH (1st tertile vs. 3rd tertile)

CRP was measured by latex immunonephelometry on a BN Prospec analyzer (Dade Behring, Newark, Delaware) [12] using Dade Behring reagents by a technician blinded to all other patient information.

The QUICKI value (measure of insulin sensitivity) and HOMA-IR values (which indicate insulin resistance) were calculated as previously described, namely:

QUICKI $=1[(\log$ insulin $)+(\log$ glucose $)]$
HOMA-IR $=($ Glucose $\times$ Insulin $) / 405$

These indexes have been shown to closely correlate with values from insulin clamp studies $[13,14]$.

\section{Statistical methods}

Linear relationships between continuous variables were summarized and tested for significance using the Spearman correlation coefficient. 
To further examine the implications of cortisol level, the sample was divided into tertiles based on the stimulated cortisol values. The first and last tertile subgroups were compared using the independent $t$-test modified, when appropriate, with the Satterthwaite adjustment.

Multivariate analyses were done using 6 models:

2 models for intra-abdominal fat and extra abdominal fat as the dependent variables while controlling for insulin, glucose, gender, age, TC, TG, and HDL.

4 models for glucose, insulin, QUICKI and HOMA as dependent variables, while controlling for intra-abdominal fat, extra abdominal fat, gender, age, TC, TG, and HDL.

P-values less than or equal to 0.05 were deemed statistically significant; no multiple-test adjustment to the p-value was done. All analyses were conducted using SAS 9.3 (SAS Institute, Inc., Cary, NC).

\section{Results}

\section{A. Simple correlations}

Cortisol suppression: All subjects responded to dexamethasone administration with suppression of cortisol below $<1.8 \mathrm{mcg} / \mathrm{dl}$, thus being ruled out for Cushing's syndrome.

Cortisol and glucose: Stimulated cortisol but not baseline (suppressed) cortisol correlated with baseline glucose $(r=0.09$, $\mathrm{p}=0.0041)$, baseline insulin $(\mathrm{r}=0.12, \mathrm{P}<0.0001)$, stimulated glucose $(\mathrm{r}=0.07, \mathrm{p}=0.01)$ and stimulated insulin $(\mathrm{r}=0.10, \mathrm{p}<<0.005)$. Cortisol was also positively correlated with HOMA and negatively with QUICKI ( $\mathrm{p}<0.005$ for both)

Cortisol and fat: Stimulated cortisol but not baseline cortisol was correlated with intra-abdominal fat $(\mathrm{r}=0.13, \mathrm{p}<0.0001)$, extra abdominal fat $(r=0.16, p<0.0001)$, abdominal height $(r=0.08, p=0.01)$, and BMI $(\mathrm{r}=0.12, \mathrm{p}<0.0001)$.

Lipids: Stimulated, but not suppressed, cortisol was positively correlated with TC $(\mathrm{r}=0.17, \mathrm{p}<<0.0001)$ TG $(\mathrm{r}=0.09, \mathrm{p}=0.001)$, LDL $(\mathrm{r}=0.11, \mathrm{p}=0.0003)$, Apo B $(\mathrm{r}=0.15, \mathrm{p}=0.0007)$, and HDL $(\mathrm{r}=0.12$, $\mathrm{p}<0.0001)$

Stimulated cortisol correlated with the Framingham scores $(r=0.17$, $\mathrm{p}<0.0001$ )

Blood pressure: Stimulated cortisol did not correlate with systolic or diastolic blood pressure ( $\mathrm{p}=\mathrm{NS}$ ).

CRP: Stimulated cortisol correlated weakly with CRP $(r=0.08$, $\mathrm{p}=0.005$ ).

There were no correlations between cortisol and baseline CAC or CAC progression.

\section{B. Analysis by tertiles of stimulated cortisol}

As seen in table 1, many parameters were associated with having stimulated cortisol in the upper tertile vs. the first tertile. Most of them are as expected except that there is also a positive correlation with HDL and again no correlation with blood pressure.

\section{Multivariate analyses}

Cortisol secretion was positively correlated with both intraabdominal fat $(p<0.0001)$ and extra abdominal fat $(p=0.01)$ after controlling for glucose, insulin, age, gender, TC, TG and HDL.

Cortisol secretion was correlated with insulin $(\mathrm{p}=0.03)$, glucose ( $\mathrm{p}=0.005)$, QUICKI $(\mathrm{p}=0.0005)$ and HOMA $(\mathrm{p}=0.0084)$ after controlling for intra-abdominal fat, extra abdominal fat, age, gender, TC, TG and HDL.

\section{Discussion}

This section of Risk Factor sub study of the St Francis Heart Study was designed to investigate the possible role of cortisol secretion, in response to $\mathrm{ACTH}$, in obesity, insulin resistance and cardiovascular risk. The metabolic presentation of Cushing's syndrome resembles an extreme form of the metabolic syndrome with increased glucose and insulin resistance and even frank diabetes, hypertension, central obesity and lipid abnormalities. It is also known that Cushing's syndrome increases the risk of CVD [15-17]. Additionally, excessive cortisol concentration due to pharmacologic use [18] or to adrenal hyperactivity [19] also results in similar metabolic derangements and elevated risk of CVD. Moreover, treatment of patients with Cushing's disease using mifepristone, a cortisol receptor antagonist, reduces serum glucose. However based on the dexamethasone suppression test none of our subjects had Cushing's syndrome. We hypothesized that even in such patients stress, mediated by excessive secretion of cortisol, and are an inducer of at least some components of the metabolic syndrome such as obesity, insulin resistance and lipid abnormalities. We used ACTH stimulation as a simulation of physiological stress and recorded the relationship of such simulated stress, as measured by stimulated cortisol secretion, with metabolic parameters that are part of the metabolic syndrome.

The result of this study did show correlations of stimulated cortisol with many features of the metabolic syndrome, including insulin resistance and hyperglycemia, central adiposity, and lipid abnormalities. Although these correlations were somewhat weak, they were all highly statistical significant and internally consistent. Furthermore when analyzing by tertiles of cortisol concentrations, the same highly significant correlations were observed. Furthermore multivariate analyses showed independent correlations of stimulated cortisol secretion with both central obesity (both intra-abdominal fat and extra abdominal fat) and insulin resistance.

There are several reasons why these correlations were not stronger. The response to stress is quite complex. The hypothalamic-pituitaryadrenal axes depend on central nervous system stimulation, causing pituitary stimulation, which in turn causes adrenal stimulation. We evaluated only the last part of this axis which probably accounts for only a fraction of the complete response, and only on one occasion. It is possible that continuous temporal measurements of cortisol secretion would demonstrate stronger correlations. Other studies which evaluated the complete axis have shown more significant correlation of the stress response with risk factors and CVD. For example, cortisol response to mental stress was correlated with the rate of incident hypertension [20] and progression of coronary calcium scores [4].

Although this study can only demonstrate correlations and not cause and effect, increased secretion of cortisol in response to ACTH was correlated with most of the parameters of the metabolic syndrome 
and the data suggests that adrenal hyper responsiveness to stress, here simulated by ACTH injection, may play at least a partial role in the metabolic abnormalities seen in the metabolic syndrome. Two exceptions were the lack of correlation with blood pressure and the positive correlation with HDL (although the correlation with TG was also positive).

From the known mechanisms of actions of cortisol, whether increased endogenously, or via pharmacological administration, it can certainly be surmised that such increase in serum cortisol would lead to increase glucose and insulin, insulin resistance, increased central adiposity, and increased lipids. Whether the increased glucose and insulin are a direct result of increased cortisol or a secondary result of increase central adiposity cannot be determined from these data. It is known that cortisol not only affects insulin resistance, but does so differentially. It has been shown that cortisol increases insulin sensitivity in adipose tissue, leading to increased glucose entry, conversion to triglycerides and fat accumulation, whereas in the muscle and liver there is decreased insulin sensitivity, leading to muscle wasting and increased glucose production [21]. This muscle loss, in turn, leads to decreased overall energy utilization and further increase in insulin resistance, setting up a vicious cycle. Our subjects demonstrated a non-pathologic (in classic terms) analogue of the situation in people with Cushing's Syndrome, who typically display many of the characteristics demonstrated in our subjects, such as increased central adiposity, insulin resistance and lipid abnormalities.

We found no correlation of cortisol secretion with blood pressure. This may be because the increased blood pressure seen in Cushing's disease is due to increased ACTH-mediated aldosterone secretion and not cortisol, and we did not measure aldosterone secretion in response to ACTH.

We also found a positive correlation with HDL. The significance of this finding is not clear. Although HDL is considered protective against CVD, several agents that raised HDL including CETP inhibitors $[22,23]$, niacin [24] and fenofibrate [25] failed to prevent CVD events. It has been speculated that the reason for these observation is that these therapies increased the wrong subset of HDL. We did not measure HDL size or composition so we do not know if this also applies in our case.

We also found that increased cortisol secretion was associated with increases of both intra-abdominal fat and extra abdominal fat, whereas it has been speculated that intra-abdominal fat is worse than extra abdominal fat. However, since our measurements where done with a single CT slice at the level of the umbilicus, both can be considered central adiposity in our case.

These findings have implications for possible ways to prevent or treat the metabolic syndrome, namely by reducing cortisol secretion or cortisol effectiveness. Four approaches are possible. One would be to utilize a block on the peripheral action of cortisol, via intracellular receptor antagonists. The utility of the latter approach in Cushing's syndrome has been demonstrated with mifepristone [26] which is now FDA approved to treat hyperglycemia in patients with Cushing's syndrome. This approach could also apply to the metabolic syndrome if increased cortisol at baseline or in response to stress is the cause for this syndrome. Other antagonists such as dexamethasone 21methylate could potentially be used. Another approach is to have a low grade chronic inhibition of cortisol synthesis with agents such as trilostane, mitotane or ketoconazole, such that normal physiology under conditions of low stress is maintained while spikes in cortisol secretion in response to stress are suppressed. A third approach would be to find ways to either reduce stress in general or inhibit ACTH secretion in response to stress. Lastly, inhibiting peripheral conversion of inactive cortisone to active cortisol decreases the overall activity of cortisol. Any of these approaches or a combination may reverse the vicious cycle described above and lead to diminished central obesity and insulin resistance, with the obvious secondary benefits of decrease progression to frank diabetes and its complications as well as decreased incidence of CVD. In summary, excessive cortisol secretion in response to $\mathrm{ACTH}$, even within the normal range, may lead to the development of the central obesity and insulin resistance.

\section{Acknowledgements}

Authors' contributions: YA and ADG designed the study, secured funding, supervised the conduct of the study, analyzed the results and wrote the manuscript. MR was in charge of the day to day conduct of the study and supervised other nurses. SP performed all statistical analyses and assisted in writing the manuscript. All authors had full access to the data. The study was funded by the St. Francis Foundation with no restriction or participation in the actual study.

The study represents valid work and was not previously presented anywhere and is not under consideration for publication elsewhere.

Yadon Arad MD, FACP, FACC is the Guarantor.

\section{Conflict of Interest}

None of the authors have any conflict of interest.

\section{References}

1. Grundy SM (2012) Pre-diabetes, metabolic syndrome, and cardiovascular risk. J Am Coll Cardiol 59: 635-643.

2. Reaven GM (2011) The metabolic syndrome: time to get off the merrygo-round? J Intern Med 269: 127-136.

3. Chanson P, Salenave S (2010) Metabolic syndrome in Cushing's syndrome. Neuroendocrinology 92 Suppl 1: 96-101.

4. Hamer M, Endrighi R, Venuraju SM, Lahiri A, Steptoe A (2012) Cortisol responses to mental stress and the progression of coronary artery calcification in healthy men and women. PLoS One 7: e31356.

5. Dimsdale JE (2008) Psychological stress and cardiovascular disease. J Am Coll Cardiol 51: 1237-1246.

6. Franke WD, Ramey SL, Shelley MC (2002) Relationship between cardiovascular disease morbidity, risk factors, and stress in a law enforcement cohort. Journal of occupational and environmental medicine / American College of Occupational and Environmental Medicine 44: 1182-9.

7. Iso H, Date C, Yamamoto A, Toyoshima H, Tanabe N, et al. (2002) Perceived mental stress and mortality from cardiovascular disease among Japanese men and women: the Japan Collaborative Cohort Study for Evaluation of Cancer Risk Sponsored by Monbusho (JACC Study). Circulation 106: 1229-36.

8. Arad Y, Newstein D, Roth M, Guerci AD (2001) Rationale and design of the St. Francis Heart Study: a randomized clinical trial of atorvastatin plus antioxidants in asymptomatic persons with elevated coronary calcification. Controlled clinical trials 22: 553-72.

9. Arad Y, Spadaro LA, Roth M, Newstein D, Guerci AD (2005) Treatment of asymptomatic adults with elevated coronary calcium scores with atorvastatin, vitamin C, and vitamin E: the St. Francis Heart Study randomized clinical trial. Journal of the American College of Cardiology 46: 166-72.

10. Arad Y, Goodman KJ, Roth M, Newstein D, Guerci AD (2005) Coronary calcification, coronary disease risk factors, C-reactive protein, and 
Citation: Arad Y, Pollack S, Roth M, Guerci AD (2015) Adrenal Cortisol Hyper-Responsiveness as a Possible Cause of Obesity, Insulin Resistance and Cardiovascular Risk. J Diabetes Metab 6: 608. doi:10.4172/2155-6156.1000608

Page 5 of 5

atherosclerotic cardiovascular disease events: the St. Francis Heart Study Journal of the American College of Cardiology 46: 158-65.

11. Kang SJ, Kim D, Park HE, Choi SH, Choi SY, et al. (2014) Visceral adipose tissue area is associated with coronary stenosis and noncalcified plaques. Int J Obes (Lond) 38: 272-278.

12. Luhr TA (2000) Use of a high-sensitivity C-reactive protein assay in evaluating cardiovascular disease risk. Am Clin Lab 19: 20-21.

13. Katsuki A, Urakawa H, Gabazza EC, Murashima S, Nakatani K, et al. (2005) Quantitative insulin sensitivity check index is a useful indicator of insulin resistance in Japanese metabolically obese, normal-weight subjects with normal glucose tolerance. Endocrine journal 52: 253-7.

14. Malita FM, Messier V, Lavoie JM, Bastard JP, Rabasa-Lhoret R, et al. (2010) Comparison between several insulin sensitivity indices and metabolic risk factors in overweight and obese postmenopausal women: a MONET study. Nutrition, metabolism, and cardiovascular diseases: NMCD 20: 173-9.

15. De Leo M, Pivonello R, Auriemma RS, Cozzolino A, Vitale P, et al. (2010) Cardiovascular disease in Cushing's syndrome: heart versus vasculature. Neuroendocrinology 92 Suppl 1: 50-54.

16. Fallo F, Famoso G, Capizzi D, Sonino N, Dassie F, et al. (2013) Coronary microvascular function in patients with Cushing's syndrome. Endocrine 43: 206-213.

17. Neary NM, Booker OJ, Abel BS, Matta JR, Muldoon N, et al. (2013) Hypercortisolism is associated with increased coronary arterial atherosclerosis: analysis of noninvasive coronary angiography using multidetector computerized tomography. The Journal of clinical endocrinology and metabolism 98: 2045-52.

18. Fardet L, Petersen I, Nazareth I (2012) Risk of cardiovascular events in people prescribed glucocorticoids with iatrogenic Cushing's syndrome: cohort study. BMJ 345: e4928.
19. Rosmond R, Björntorp P (2000) The hypothalamic-pituitary-adrenal axis activity as a predictor of cardiovascular disease, type 2 diabetes and stroke. J Intern Med 247: 188-197.

20. Hamer M, Steptoe A (2012) Cortisol responses to mental stress and incident hypertension in healthy men and women. J Clin Endocrinol Metab 97: E29-34.

21. Whorwood CB, Donovan SJ, Flanagan D, Phillips DI, Byrne CD (2002) Increased glucocorticoid receptor expression in human skeletal muscle cells may contribute to the pathogenesis of the metabolic syndrome. Diabetes 51: 1066-75.

22. von Eckardstein A (2010) Implications of torcetrapib failure for the future of HDL therapy: is HDL-cholesterol the right target? Expert Rev Cardiovasc Ther 8: 345-358.

23. Lim GB (2011) Coronary artery disease: Dalcetrapib safely raises HDLcholesterol level in the phase IIb dal-PLAQUE trial. Nat Rev Cardiol 8: 610 .

24. Teo KK, Goldstein LB, Chaitman BR, Grant S, Weintraub WS, et al. (2013) Extended-Release Niacin Therapy and Risk of Ischemic Stroke in Patients With Cardiovascular Disease: The Atherothrombosis Intervention in Metabolic Syndrome With Low HDL/High Triglycerides: Impact on Global Health Outcome (AIM-HIGH) Trial. Stroke; a journal of cerebral circulation.

25. Ismail-Beigi F, Craven T, Banerji MA, Basile J, Calles J, et al. (2010) Effect of intensive treatment of hyperglycaemia on microvascular outcomes in type 2 diabetes: an analysis of the ACCORD randomised trial. Lancet 376: 419-30

26. Carroll T, Findling JW (2012) The use of mifepristone in the treatment of Cushing's syndrome. Drugs Today (Barc) 48: 509-518. 